\title{
PSYCHOLOGICA
}

\section{Perfis de adaptação psicológica na criança com asma}

Autor(es): $\quad$ Lima, Lígia; Guerra, Marina Prista; Lemos, Marina Serra de

Publicado por: Imprensa da Universidade de Coimbra

URL

persistente: URI:http://hdl.handle.net/10316.2/3442

DOI: $\quad$ DOI:http://dx.doi.org/10.14195/1647-8606_52-1_5

Accessed : $\quad$ 26-Apr-2023 15:56:55

A navegação consulta e descarregamento dos títulos inseridos nas Bibliotecas Digitais UC Digitalis, UC Pombalina e UC Impactum, pressupõem a aceitação plena e sem reservas dos Termos e Condições de Uso destas Bibliotecas Digitais, disponíveis em https://digitalis.uc.pt/pt-pt/termos.

Conforme exposto nos referidos Termos e Condições de Uso, o descarregamento de títulos de acesso restrito requer uma licença válida de autorização devendo o utilizador aceder ao(s) documento(s) a partir de um endereço de IP da instituição detentora da supramencionada licença.

Ao utilizador é apenas permitido o descarregamento para uso pessoal, pelo que o emprego do(s) título(s) descarregado(s) para outro fim, designadamente comercial, carece de autorização do respetivo autor ou editor da obra.

Na medida em que todas as obras da UC Digitalis se encontram protegidas pelo Código do Direito de Autor e Direitos Conexos e demais legislação aplicável, toda a cópia, parcial ou total, deste documento, nos casos em que é legalmente admitida, deverá conter ou fazer-se acompanhar por este aviso. 


\section{NÚMERO 52}

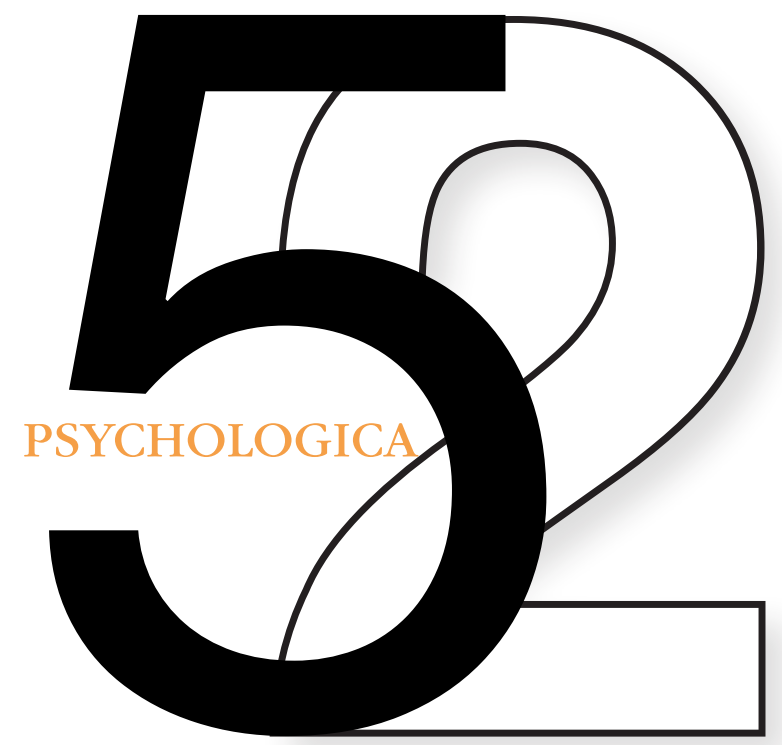

VOLUME I

IMPRENSA DA UNIVERSIDADE DE COIMBRA

FACULDADE DE PSICOLOGIA E DE CIÊNCIAS DA EDUCAÇÃO DA UNIVERSIDADE DE COIMBRA 


\title{
Perfis de adaptação psicológica na criança com asma
}

\author{
Lígia Lima', Marina Prista Guerra² \& Marina Serra de Lemos $^{3}$
}

Este estudo tem como objectivos caracterizar sob um ponto de vista psicológico as crianças com asma e suas famílias e identificar diferentes perfis de adaptação. A amostra consistiu em 89 crianças, de ambos os sexos e com idades entre os 8 e os 12 anos e com asma. Relativamente à criança avaliaram-se o temperamento, inteligência, auto-estima e coping. Quanto à família foram avaliados o tipo de agregado familiar, o humor da mãe e as alterações e interferências causadas pela asma nas rotinas familiares. Como indicadores de adaptação foram avaliados sintomas de ansiedade/depressão, as habilidades sociais e a qualidade de vida.

Os resultados encontrados sugerem que as crianças com asma, enquanto grupo, apresentam características que apontam maiores dificuldades de adaptação psicológica. No entanto, os resultados da análise de clusters, revelaram dois perfis distintos de adaptação das crianças com asma sugerindo que apesar de a asma representar uma situação de risco parece possivel lidar favoravelmente com a doença.

PALAVRAS-CHAVE: Asma, Perfis de adaptação psicológica, Análise de clusters.

\section{Introdução}

A investigação recente tem vindo a sugerir que a maioria das crianças com asma e suas famílias se conseguem adaptar a esta doença sem grandes dificuldades (Barros, 2003), mas também evidencia que a asma pediátrica constitui um risco em termos de adaptação e desenvolvimento psicológicos (Bacharier, Boner, Carlsen, Eigenmann, Frischer et al. 2008; McQuaid, Kopel \& Nassau, 2001; Thompson \& Gustafson, 1996). Existe já muita investigação sobre a vivência psicológica da doença asmática, mas persiste ainda um grande desconhecimento acerca dos processos envolvidos. Existe uma grande inconsistência de resultados e, na maior parte das vezes, a problemática tem sido estudada no âmbito de uma abordagem centrada nas variáveis, a qual não considera que cada factor estudado só adquire significado na configuração de outras variáveis que caracterizam o indivíduo

\footnotetext{
1 Escola Superior de Enfermagem do Porto - lígia@esenf.pt

2, 3 Faculdade de Psicologia e Ciências da Educação da Universidade do Porto.
} 
(Smeekens, Riksen-Walraven \& Bakel, 2008). Em alternativa, neste estudo procurámos fazer uma análise mais centrada no indivíduo, com vista a identificar diferentes perfis de adaptação à asma, compostos pelas diferentes combinações entre os factores em estudo. Foram seleccionadas as variáveis que, de acordo com uma revisão da literatura, estariam mais directamente implicadas no processo de adaptação da criança com doença crónica em geral e da asma em particular, quer por funcionarem como área de expressão das implicações da vivência de doenças deste tipo nestas idades, quer por actuarem como factores que podem agravar ou atenuar os efeitos de se viver com este tipo de patologia. Decidimos ainda estudar variáveis relativas quer à criança, quer à sua família e quer ainda à sua doença, por considerarmos que a partir da compreensão da inter-relação entre elas poderemos dar um contributo adicional para a compreensão do nosso objecto de estudo, a adaptação psicológica da criança com asma.

De entre as variáveis relacionadas com a criança, incluímos em primeiro lugar o temperamento. Há autores que defendem que as crianças com asma se distinguem das crianças saudáveis em termos de temperamento, independentemente de se considerar que essas diferenças são prévias ou subsequentes ao desencadear da doença (Liu \& Wan, 2001), mas mais frequentemente, a investigação tem vindo a demonstrar que os traços temperamentais funcionam como variáveis intermédias no processo que descreve o impacto de uma doença crónica na adaptação psicológica da criança (Benchell \& Glasgow, 1997; Wallander \& Varni, 1998). Também a inteligência tem sido apontada como um factor protector para a adaptação psicológica (Masten, Morison, Pellegrini \& Tellegen, 1992). É ainda a nivel da inteligência, que alguns autores consideram que é possivel encontrar diferenças entre as crianças saudáveis e as crianças com asma, em parte como consequência de estas últimas terem que optar por actividades de lazer mais do tipo sedentário, o que Ihes possibilitaria desenvolver mais as competências do tipo verbal (French, Cristie \& West, 1984). Segundo Trad e Greenblatt (1990 cit in Sharp \& Cowie, 1998) uma baixa auto-estima constitui uma das expressões mais comuns da exposição a um stressor do tipo crónico, e uma boa auto-estima constitui um factor protector geral segundo a literatura sobre o risco e resiliência (Rutter, 1992). Por estas razões, a auto-estima foi outra das variáveis avaliadas.

Incluiu-se ainda a utilização de estratégias de coping, dado que as crianças que possuem um amplo repertório de estratégias de coping são frequentemente resilientes face a situações de risco em geral e face a situações de doença crónica em particular (Ryan-Wenger, Sharrer \& Wynd, 2000).

Um outro grupo de variáveis que considerámos fundamental estudar no sentido de compreender a adaptação das crianças com asma, diz respeito à sua famí- 
lia mais restrita. A escolha da variável tipo de agregado familiar decorreu do pressuposto que uma estrutura familiar nuclear não intacta poderá constituir um factor de risco para a adaptação emocional da criança com doença crónica (Brown, Wiener, Kupst, Brennan, Behrman, Compas et al. 2008). A saúde mental da mãe constitui um outro factor que tem vindo a ser sistematicamente apontado como estando associada à adaptação psicológica das crianças em geral (Pauli-Pot, Darui \& Beckman, 1999) assim como das crianças com asma (Kaugars, Klinnert \& Bender, 2004), razões que nos levaram a incluir também a variável humor da mãe no nosso estudo. Por fim, escolhemos ainda uma variável relacionada com o impacto da asma na organização da vida familiar, na tentativa de avaliar em que medida a asma constituía um stressor para a família da criança, o que indirectamente poderia reflectir-se também a nível da adaptação da criança (Thompson \& Gustafson, 1996).

A adaptação psicológica das crianças com asma foi conceptualizada de forma multidimensional, na tentativa de ser a mais compreensiva possivel. Considerou-se assim a adaptação enquanto competência, ou seja, em termos de um funcionamento bem sucedido e apropriado para o nível de desenvolvimento da criança, tendo sido privilegiado o funcionamento no meio escolar, mais especificamente as habilidades sociais da criança. A qualidade da adaptação da criança com doença asmática foi também avaliada através da presença de sintomas de ansiedade/ depressão, dado que a literatura mostra que este tipo de sintomas constitui os mais frequentemente apresentados pelas crianças com doença crónica (Barros, 2003).

Por fim, a adaptação foi ainda avaliada através da qualidade de vida geral percebida pelas próprias crianças. No domínio geral da Psicologia pediátrica, e em especial, no estudo da adaptação psicológica à asma, esta também foi já identificada como sendo uma variável que exprime o grau de maior ou menor sucesso no processo de gestão da doença (Everhart \& Fiese, 2008; Vinson, 2002).

Foram traçados dois objectivos para este estudo. Um primeiro pretendeu caracterizar sob um ponto de vista psicológico as crianças com asma e suas famílias. Foi nosso objectivo conhecer melhor as características destas crianças com vista a podermos avaliar se de facto possuem aspectos que as identificam enquanto grupo, ou se pelo contrário, existe uma tal diversidade que torna difícil diferenciá-las pelo facto de sofrerem de asma. Como segundo objectivo, pretendemos estudar a variabilidade intra-grupo, procurando identificar diferentes perfis de adaptação à asma, compostos por diferentes combinações entre as variáveis em estudo. Utilizámos para tal, o método de análise de clusters, partindo do pressuposto de que este método nos permitirá identificar formas diferentes de lidar com a doença e levantar hipóteses relativamente à sua maior ou menor adaptabilidade. 


\section{Método}

\subsection{Participantes}

A amostra deste estudo é do tipo sequencial e de conveniência. Participaram 89 crianças que frequentavam as consultas de Imuno-alergologia Pediátrica de um Hospital Central e a consulta de saúde familiar dum Centro de Saúde do Distrito do Porto, com asma do tipo intermitente, persistente ligeiro ou persistente moderado. A classificação do tipo de asma foi realizada de acordo com o diagnóstico feito durante consulta que precedeu a realização da entrevista e seguindo os critérios da "Global Initiative for Asthma [G.I.N.A] criteria" (1995). A decisão de eliminar os casos de asma persistente grave prendeu-se ainda com o facto de serem mais susceptíveis à psicopatologia e de, segundo a opinião dos médicos assistentes, se diferenciarem bastante dos casos dos restantes graus de gravidade, quer relativamente à frequência e gravidade das crises, interferência dos sintomas no funcionamento da criança e do tipo de medicação administrada. Pretendemos com estes critério encontrar uma amostra relativamente homogénea em termos clínicos. Assim 6 (6,7\%) das crianças da amostra possuíam asma do tipo intermitente, $54(60,7 \%)$ do tipo persistente ligeiro e 29 (32,6\%) do tipo persistente moderado.

As crianças da amostra possuíam entre 8 e 12 anos de idade, com uma média de 9,71. Destas $58(65,2 \%)$ eram do sexo masculino e as restantes $31(34,8 \%)$ do sexo feminino. A maior parte das crianças estava acompanhada pela mãe $(86,5 \%)$ no momento da consulta e da entrevista, enquanto as restantes estavam acompanhadas pelo pai (4,5\%), pela avó (7,8\%), ou por ambos os pais (pai e mãe) $(1,1 \%)$.

\subsection{Instrumentos}

Neste estudo foram utilizados vários instrumentos, para além de um questionário construído especificamente para este estudo e a ser preenchido pela mãe ou cuidador principal da criança, visando obter os seguintes dados: identificação e caracterização socio-demográfica individual e familiar da criança, mais concretamente, idade, sexo, ano de escolaridade, profissão pais, composição agregado familiar; tipo de agregado familiar (intacto ou não); alterações e interferências na vida familiar causadas pela asma e humor/boa disposição da mãe. Os instrumentos utilizados, cujas propriedades psicométricas foram previamente estudadas, são os seguintes:

Teste das Matrizes Progressivas Coloridas de Raven - é um instrumento de avaliação da inteligência, mais especificamente da inteligência não verbal. Este instrumento está aferido para a população portuguesa por Simões (2000), tendo revelado valores muito satisfatórios (alphas $\geq 0,80$ ). 
Versão Portuguesa do School-age Temperament Inventory [SATI] de (Lima, Lemos \& Guerra, 2010) é um questionário de auto-registo destinado a pais de crianças com idades compreendidas entre os 8 e os 11 anos de idade. $O$ inventário permite avaliar quatro dimensões do temperamento: reactividade negativa (negative reactivity), persistência de tarefa (task persistance), sociabilidade ${ }^{4}$ (approach/ withdrawal) e actividade (activity). Os coeficientes alpha de Cronbach para as quatro subescalas são de $0,87,0,84,0,82$ e 0,77, respectivamente.

Subescala de Avaliação da Auto-estima Global - faz parte de um instrumento de Susan Harter - The Self-perception Profile for Children (1985) e procura avaliar o sentimento geral que a criança possui acerca de si e da sua experiência de vida. A adaptação para a população portuguesa foi realizada por Isabel Sá (1997 cit in Ferreira, 2000) e os valores da análise factorial e da validade de constructo revelaram-se satisfatórios.

Versão Portuguesa do Schoolagers Coping Strategies Inventory (SCSI) de (Lima, Lemos e Guerra, 2002) é um instrumento de auto-registo, destinado a crianças com idades compreendidas entre os 8 e os 12 anos de idade, que avalia a percepção acerca da sua utilização de estratégias de coping (em duas sub-escalas frequência e eficácia) durante um acontecimento stressante pessoalmente definido pela criança (Ryan-Wenger, 1990). Possui uma estrutura factorial de 3 dimensões correspondentes a três tipos de estratégias: de distracção cognitivo comportamental, de acting-out e activas. Os alphas de Cronbach variam entre 0,72 e 0,78, com excepção da dimensão relativa às estratégias activas ( $\alpha=0,50 / 0,53$ para as escalas de frequência e eficácia, respectivamente).

Escala para a Avaliação de sintomas de Ansiedade/Depressão na Criança - conjunto de 14 itens adoptados do Child Behavior Checklist (CBCL) de Achenbach (1991) que contêm descrições de comportamentos depressivos ou ansiosos da criança. Calculámos a consistência interna dos itens da escala ansiedade/depressão através do cálculo do alpha de Cronbach, tendo sido encontrado um valor satisfatório, de 0,78 , confirmando a precisão da escala.

Versão Portuguesa da Escala de Avaliação de Habilidades Sociais de Gresham e Elliot (1990), por Lemos e Meneses (2002). A escala de habilidades sociais faz parte do Social Skills Rating System de Gresham e Elliot (1990) e destina-se a ser preenchida pelo professor da criança. Os valores de alpha das subescalas são de $\alpha=0,93$ para a escala total de habilidades sociais; $\alpha=0,90$ para a subescala de auto-controlo, $\alpha=0,91$ para a subescala de cooperação e $\alpha=0,93$ para a subescala de assertividade.

\footnotetext{
4 Dado que esta subescala é inversamente cotada, na verdade o valor exprime o grau de retraimento da criança.
} 
A Versão Portuguesa da Pediatric Quality of Life Inventory 4.0 - (PedsOL) por (Lima, Guerra e Lemos, 2009) constitui uma forma modular de avaliação da qualidade de vida relacionada com a saúde e neste estudo utilizámos a Escala Genérica de auto-registo para crianças entre os 8 e 12 anos de idade. A versão portuguesa do instrumento integra menos 3 itens do que a PedsOL original, no entanto a análise factorial replica os factores propostos pela versão original. Relativamente ao estudo da fidelidade do instrumento, a consistência interna da escala geral é francamente satisfatória $(\alpha=0,85)$, confirmando a precisão da escala. Os coeficientes obtidos para as subescalas física $(\alpha=0,74)$ e psicossocial $(\alpha=0,81)$ são também elevados. Já os valores de alpha obtidos para as quatro dimensões avaliadas pela escala (funcionamento físico, funcionamento emocional, funcionamento social e funcionamento escolar) variam entre o,67 e o,74.

\subsection{Procedimento}

Após a aprovação da Comissão de ética do Hospital e da Direcção do Centro de Saúde, foram os médicos assistentes (alergologistas ou médicos de família) que fizeram o primeiro contacto com as crianças e acompanhantes (na maior parte das situações a mãe) e que solicitaram a sua colaboração, com carácter voluntário. A aplicação dos instrumentos foi feita durante uma entrevista realizada em simultâneo à criança e mãe (ou acompanhante), que se iniciava com uma breve apresentação do estudo, incluindo uma referência aos objectivos e metodologias. Seguia-se o pedido de preenchimento (por escrito) do formulário do consentimento informado por parte do cuidador.

No final da entrevista era pedida autorização para envio de questionários à professora ou directora de turma, que era mais uma vez precedida de uma explicação da finalidade destes instrumentos. É de referir que apenas 64 dos professores das 89 crianças avaliadas devolveram o formulário devidamente preenchido, pelo que nem todos os sujeitos da nossa amostra serão descritos e avaliados relativamente às suas habilidades sociais.

\section{Resultados}

\subsection{Caracterização das crianças com asma e suas famílias segundo as variáveis em estudo}

Com vista a caracterizar as crianças participantes e suas famílias relativamente às variáveis em estudo foi realizada uma primeira análise descritiva, cujos resultados estão indicados no Quadro 1. Para facilitar a interpretação dos dados foi 
introduzida no quadro informação relativa a dados normativos obtidos com crianças saudáveis.

Quadro 1. Caracterização da amostra relativamente às variáveis em estudo.

\begin{tabular}{|c|c|c|c|c|}
\hline & \multicolumn{2}{|c|}{$\begin{array}{l}\text { Amostra de crianças } \\
\text { com asma }\end{array}$} & \multicolumn{2}{|c|}{$\begin{array}{l}\text { Valores de referência } \\
\text { obtidos com crianças } \\
\text { saudáveis }\end{array}$} \\
\hline & Média & DP & Média & DP \\
\hline \multicolumn{5}{|l|}{ Temperamento } \\
\hline - Reactividade Negativa (RN) & 3,18 & 0,79 & 2,76 & 0,66 \\
\hline - Persistência na Tarefa (PT) & 3,41 & 0,79 & 3,75 & 0,73 \\
\hline - Sociabilidade (Soc) & 2,80 & 0,89 & 2,39 & 0,68 \\
\hline - Actividade (Act) & 3,13 & 0,87 & 2,75 & 0,74 \\
\hline Auto-estima global & 3,01 & 0,56 & 3,07 & 0,63 \\
\hline \multicolumn{5}{|l|}{ Coping-frequência } \\
\hline - Estratégias de distrac. cog. e comp. & 14,21 & 5,25 & 15,14 & 5,68 \\
\hline - Estratégias de acting out & 2,71 & 2,14 & 3,43 & 2,92 \\
\hline - Estratégias Activas & 7,78 & 2,87 & 8,96 & 2,70 \\
\hline - Total & 24,70 & 7,86 & 27,70 & 8,01 \\
\hline \multicolumn{5}{|l|}{ Coping - eficácia } \\
\hline - Estratégias de distrac. cog. e comp. & 17,08 & 5,26 & 17,07 & 5,57 \\
\hline - Estratégias de acting out & 2,89 & 2,20 & 3,41 & 2,51 \\
\hline - Estratégias Activas & 12,02 & 3,41 & 11,99 & 2,73 \\
\hline - Total & 31,99 & 8,33 & 32,78 & 8,07 \\
\hline \multicolumn{5}{|l|}{ Qualidade de Vida } \\
\hline - Funcionamento Físico & 71,21 & 20,96 & 83,50 & 14,77 \\
\hline - Funcionamento Emocional & 65,51 & 19,28 & 73,34 & 16,70 \\
\hline - Funcionamento Social & 81,61 & 17,58 & 84,57 & 15,11 \\
\hline - Funcionamento Escolar & 71,29 & 16,35 & 78,16 & 15,85 \\
\hline - Subtotal Saúde Física & 71,21 & 20,96 & 83,50 & 14,77 \\
\hline - Subtotal Saúde Psicossocial & 72,17 & 13,01 & 78,23 & 12,91 \\
\hline - Total & 71,88 & 13,72 & 79,81 & 12,07 \\
\hline Habilidades sociais & 39,78 & 9,73 & & \\
\hline Ansiedade/depressão & 9,13 & 4,58 & & \\
\hline \multicolumn{5}{|l|}{ Inteligência: } \\
\hline - 8 anos & 25,08 & 5,90 & 20,46 & 5,72 \\
\hline - 9 anos & 27,71 & 5,99 & 23,04 & 6,11 \\
\hline - 10 anos & 27,59 & 4,72 & 25,54 & 5,70 \\
\hline - 11 anos & 30,26 & 5,02 & 27,34 & 5,04 \\
\hline - 12 anos & 30,73 & 3,58 & - & - \\
\hline
\end{tabular}

\subsection{Estudo de perfis de adaptação psicossocial à asma ligeira e moderada} Como forma de estudar a variabilidade intra-grupo no processo de adaptação psicossocial à asma, procurámos encontrar distintos subgrupos de crianças, com base em determinados perfis de adaptação, que foram definidos através da semeIhança (proximidade) das características avaliadas, ou seja, através do método 
de análise de clusters (Borgen \& Barnett, 1987). Realizaram-se ainda diferenças de médias relativas às variáveis não incluídas nos perfis. Relativamente às habilidades sociais e à realização académica apenas possuíamos dados para 63 e 64 sujeitos respectivamente, pelo que não puderam ser incluídas na análise de clusters. A gravidade da asma também não foi incluída na determinação dos clusters, uma vez que a nossa amostra possuía um número muito reduzido de crianças com asma do tipo intermitente.

Para a identificação de perfis de adaptação, foi realizada uma série de análises de Clusters (com estandartização dos factores). A escolha do número de clusters foi realizada através de uma técnica de análise de clusters exploratória, a técnica hierárquica aglomerativa que, segundo a literatura, constitui uma forma fiável de identificar o número de clusters existente nos dados (que é utilizado posteriormente na análise $K$-means) na ausência de uma estrutura teórica subjacente que nos indique este dado (Borgen \& Barnett, 1987). Para a análise hierárquica seleccionámos o método Ward como procedimento para a aglomeração dos clusters (Borgen \& Barnett, 1987). A partir da análise do dendograma, e do quadro de aglomeração, concluímos que a solução de dividir a amostra em dois clusters era a mais adequada. Ao avaliar o quadro de aglomeração, a comparação entre os coeficientes para uma solução de 2 clusters e de 3 clusters revelou um aumento significativo no valor da medida de distância entre as duas, indicando assim uma solução de dois factores. Uma vez que diferentes métodos de aglomeração não dão necessariamente origem às mesmas soluções (Pestana \& Gageiro, 2000), para testarmos a robustez da solução escolhida, fizemos também a análise dos dados utilizando o método do Average Linkage, do qual resultou também o agrupamento dos sujeitos em dois perfis, confirmando os resultados obtidos anteriormente com o método Ward. Por fim, realizámos uma análise de clusters confirmatória, através do método $K$-means que distribuiu sucessivamente e a partir de uma série de iterações, os casos pelos clusters, até à solução final, de todos os sujeitos ficarem distribuídos em dois perfis distintos. As médias e os desvios-padrão das variáveis estudadas, para cada um dos dois agrupamentos obtidos, apresentam-se no Quadro 2.

Quadro 2. Médias e desvios padrão das variáveis em estudo para cada agrupamento de análise de clusters

\begin{tabular}{|c|c|c|c|c|c|c|c|}
\hline & $\begin{array}{l}\text { Cluster } \\
\mathrm{N}=34\end{array}$ & & $\begin{array}{l}\text { Cluster } 2 \\
\mathrm{~N}=52\end{array}$ & & & & \\
\hline & Média & D.P. & Média & D.P. & $\begin{array}{l}\text { Média } \\
\text { Global }\end{array}$ & $\mathrm{F}$ & Sig \\
\hline Depressão/ansiedade & 12,56 & 4,15 & 7,06 & 3,46 & 9,23 & 44,32 & 0,000 \\
\hline \multicolumn{8}{|l|}{ Temperamento: } \\
\hline Reactividade neg & 3,87 & 0,54 & 2,72 & 0,57 & 3,18 & 87,03 & 0,000 \\
\hline Persistência tarefa & 2,87 & 0,70 & 3,77 & 0,63 & 3,41 & 38,17 & 0,000 \\
\hline Sociabilidade & 3,08 & 0,89 & 2,61 & 0,86 & 2,80 & 5,96 & 0,017 \\
\hline
\end{tabular}




\begin{tabular}{llllllll}
\hline Actividade & 3,62 & 0,84 & 2,81 & 0,74 & 3,13 & 22,55 & 0,000 \\
\hline Estratégias de Coping: & & & & & & & \\
\hline Frequência & 28,56 & 7,34 & 22,58 & 7,20 & 24,94 & 13,82 & 0,000 \\
\hline Eficácia & 35,50 & 7,72 & 30,13 & 7,88 & 32,26 & 9,69 & 0,003 \\
\hline Qualidade de Vida & 65,59 & 7,72 & 75,10 & 11,36 & 71,34 & 11,21 & 0,001 \\
\hline Auto-estima & 2,83 & 0,62 & 3,11 & 0,50 & 3,00 & 5,44 & 0,022 \\
\hline Inteligência & 27,53 & 5,23 & 28,00 & 5,63 & 27,81 & 0,152 & 0,698 \\
\hline
\end{tabular}

Do conjunto de variáveis estudadas, as que contribuíram para discriminar os clusters, são por ordem decrescente, reactividade negativa, ansiedade/depressão, persistência na tarefa, actividade, frequência das estratégias de coping, qualidade de vida, eficácia das estratégias de coping, sociabilidade e auto-estima. A variável inteligência não contribuiu para diferenciar a classificação nos clusters. O cluster/perfil 1 caracteriza $40 \%$ dos elementos da amostra $(\mathrm{N}=34)$. As crianças com asma, classificadas neste agrupamento, apresentam os valores mais elevados nos itens referentes à depressão/ansiedade. Relativamente ao temperamento, este perfil inclui as crianças com os valores mais elevados de reactividade negativa, actividade e sociabilidade (o que significa retraimento, visto que esta dimensão é inversamente cotada) e os valores mais baixos de persistência na tarefa. Relativamente às estratégias de coping, as crianças caracterizam-se por apresentar os valores mais elevados quer relativamente à frequência quer à eficácia. Por fim, este perfil também caracteriza as crianças com pior qualidade de vida e valores mais baixos de auto-estima.

O Cluster/perfil 2 caracteriza $60 \%$ das crianças da amostra $(\mathrm{N}=52)$. Pertencem a este agrupamento as crianças com valores mais baixos de depressão/ ansiedade. No que diz respeito ao temperamento, este perfil caracteriza as crianças com valores mais baixos de reactividade negativa, e actividade e valores mais elevados de persistência na tarefa e sociabilidade (índice mais baixo na respectiva escala). Em termos de estratégias de coping, as crianças deste perfil caracterizam-se por uma menor frequência e eficácia na utilização das mesmas. Por fim, o perfil de adaptação à asma definido por este agrupamento é caracterizado por valores mais elevados de qualidade de vida e de auto-estima.

Com vista a investigar possíveis diferenças entre os sujeitos em cada um dos dois clusters em função das variáveis sócio-demográficas fizemos análises de Qui-quadrado. Não foram encontradas diferenças significativas em nenhuma das variáveis. Com o objectivo de averiguar possíveis diferenças entre as crianças pertencentes a cada um dos dois perfis relativamente às restantes variáveis estudadas e que não foram incluídas na determinação dos clusters, procedeu-se a análises de diferenças de médias utilizando diferentes métodos de acordo com o tipo de variáveis em estudo. 
Relativamente às Habilidades Sociais realizamos um teste $T$ dos resultados nos dois clusters e verificámos que existe uma diferença significativa ( $t=-2,58, p<0,05)$ nas médias das crianças distribuídas pelos dois clusters relativamente à escala habilidades sociais, isto é, as crianças do cluster $2(M=42,29)$ possuem valores mais elevados do que as crianças pertencentes ao cluster $1(M=36,12)$. Quanto à variável agregado familiar, trata-se de uma variável do tipo nominal e para facilitar a análise foi recodificada em duas categorias, ou seja agregado familiar intacto (constituído por pai e mãe e irmãos ou outros familiares) e agregado familiar não intacto (caracterizado pela ausência de uma ou as duas figuras parentais e constituidos por outros familiares como avós, tios ou outros). Procedeu-se então ao cálculo do Qui-quadrado $\chi^{2}$ tendo-se verificado diferenças significativas $\left(\chi^{2}=\right.$ $4,285, p<0,05)$ relativamente ao tipo de agregado de familiar da criança em função do seu cluster de pertença É no cluster 1 que existe maior percentagem de crianças com agregado familiar não intacto $(26,5 \%)$, visto que no cluster 2 , esta percentagem é significativamente menor (9,6\%).

Relativamente à variável disposição, energia da mãe, através do teste T verificouse que não existe diferença entre as médias das crianças pertencentes aos dois clusters. Finalmente e com o objectivo de averiguar a existência de diferenças entre os sujeitos dos dois clusters relativamente ao grau de gravidade da doenças asmática, realizamos um teste de Mann-Witney, tendo-se verificado a existência de uma diferença estatística altamente significativa $(U=0,000, p<0,001)$, isto é, no cluster 1 existe um menor número de crianças com asma do tipo ligeira e um maior número de crianças com gravidade do tipo moderada relativamente ao cluster 2 .

No Quadro 3 apresentamos a distribuição dos sujeitos pelos clusters de pertença em função da variável grau de gravidade da asma. Conforme se pode observar as crianças com asma do tipo persistente moderada (Grau 2) estão sobretudo no cluster 1.

Quadro 3. Distribuição dos sujeitos nos perfis de pertença de acordo com o tipo de gravidade da asma

\begin{tabular}{llll}
\hline & $\mathrm{N}$ & $\begin{array}{l}\text { Cluster 1 } \\
\mathrm{N}=34 \\
\mathrm{~N}(\%)\end{array}$ & $\begin{array}{l}\text { Cluster 2 } \\
\mathrm{N}=52 \\
\mathrm{~N}(\%)\end{array}$ \\
\hline $\begin{array}{lll}\text { Grau de gravidade da asma } \\
\text { Grau 0 - intermitente }\end{array}$ & 5 & $3(8,8)$ & $2(3,8)$ \\
Grau 1 - persistente ligeira & 52 & $14(41,2)$ & $38(73,1)$ \\
Grau 2 - persistente moderada & 29 & $17(50,0)$ & $12(23,1)$ \\
TOTAL & 86 & $86(100)$ & $86(100)$ \\
\hline
\end{tabular}




\section{Discussão dos resultados}

A análise descritiva da amostra por si só parece indicar que, ainda enquanto grupo, as crianças com asma parecem caracterizar-se por um certo tipo de atributos pessoais, que as colocam numa eventual situação de maior vulnerabilidade em termos de adaptação psicológica. Em termos de temperamento, os resultados encontrados confirmam estudos anteriores, nomeadamente que as crianças com asma se caracterizam por um nível mais elevado de reactividade negativa, de retraimento (Liu \& Wan, 2001) e de actividade e por resultados menos elevados de persistência na tarefa (Kim, Ferrara \& Chess, 1980). Quanto à variável inteligência, as crianças apresentaram resultados ligeiramente superiores ao esperado, o que também é segundo alguns autores, comum nas crianças com asma (Gustastadt, 1989 cit in French et al., 1984). Relativamente ao coping e tal como Lemanek e Hood (1999) sugerem, também verificamos que em geral, as crianças com asma relatavam utilizar menos frequentemente estratégias de coping quando confrontadas com situações potencialmente stressantes.

Ainda no que diz respeito a dois dos indicadores de adaptação, especificamente à ansiedade/depressão e qualidade de vida, as crianças com asma apresentaram globalmente resultados inferiores aos da população geral. Na literatura é esperado que a qualidade de vida geral das crianças diminua quando estas são confrontadas com uma doença crónica (Levi \& Drotar, 1998), como por exemplo a asma (Vinson, 1996) e de facto, comparando os resultados obtidos pelas crianças que constituíram a amostra do nosso estudo com os resultados da amostra saudável (Lima et al., 2009), pudemos verificar que as crianças com asma possuem valores inferiores nos índices de qualidade de vida aos obtidos pelas crianças saudáveis.

Relativamente ao indicador de ansiedade/depressão, segundo Achenbach (1991) os resultados médios de referência para a análise do conjunto de itens destinados a avaliar a presença de sintomas de ansiedade/depressão são de 7,9 para crianças entre os 4 e os 11 anos referenciadas para acompanhamento clínico e de 3,1 para crianças do mesmo grupo etário, não referenciadas clinicamentes. O valor médio encontrado para a nossa amostra situa-se acima destes dois valores, ou seja, é ligeiramente superior à média das crianças referenciadas para acompanhamento clínico mas ultrapassa em muito o valor das crianças pertencentes ao grupo normativo. No entanto, os valores propostos por Achenbach são provenientes da população americana e por isso servem apenas como uma possivel referência à análise das nossas respostas na ausência de uma norma portuguesa.

Relativamente ao estudo de perfis de adaptação e através do método de análise de clusters verificámos que existiam dois perfis de adaptação resultantes das rela-

5 Estes valores dizem respeito a crianças do sexo masculino e os valores do sexo feminino são ligeiramente superiores, isto é, de 3,4 para crianças não referenciadas e de 8,5 para crianças referenciadas clinicamente. 
ções entre variáveis em estudo, o que vem de encontro à literatura e investigação mais recente, que enfatiza a variabilidade intra-grupo na adaptação psicológica de crianças com doença crónica, em particular com asma (Barros, 2003). A caracterização das crianças agrupadas em cada um dos perfis foi complementada com um estudo de diferenças de médias relativamente a variáveis socio-demográficas e às variáveis que não foi possivel incluir na determinação dos clusters, por razões já expostas anteriormente. O primeiro cluster, considerado como sendo o perfil de menor adaptabilidade é constituído pelas crianças que em termos temperamentais apresentaram valores médios mais elevados de reactividade negativa, sociabilidade (significando na verdade retraimento) e actividade e valores menos elevados de persistência na tarefa. Embora não existam ainda normas para a população portuguesa, a conjugação destas características temperamentais sugere que estas crianças possuem um tipo de temperamento próximo do que McClowry (2002) denominou de desafiante e que outros autores referem como sendo dificil (Thomas \& Chess, 1977 cit in McClowry, 2002). Tendo em conta que o temperamento pode ser um factor associado aos problemas comportamentais que as crianças apresentam ao longo do seu desenvolvimento (Carranza \& Salinas, 2003), os nossos resultados poderão significar que estas crianças possuem características temperamentais menos favoráveis ao desenvolvimento de uma boa adaptação psicológica, face à vivência de uma doença crónica. Como vimos, a asma pediátrica constitui uma doença crónica, que mesmo nos casos menos graves, obriga a cumprir um certo número de tarefas não-normativas que podem constituir fontes de stress para a criança, tais como, o evitamento de certo tipo de actividades que podem funcionar como desencadeantes de crises, o cumprimento regular e sistemático de terapêuticas medicamentosas e um contacto regular com profissionais de saúde que asseguram o controlo da doença (por exemplo, no caso de a criança fazer imunoterapia). Todas estas situações se afigurarão como mais complicadas de gerir e por isso mais passiveis de causar mal-estar se, à partida, a criança já não tem muita facilidade em se adaptar a situações novas e a contactar com pessoas que não conhece, se reage mais negativamente às situações de frustração e se não possui grande grau de motivação para cumprir tarefas e responsabilidades que lhe são atribuídas.

O valor médio da auto-estima global das crianças que integram este perfil é inferior ao das crianças do cluster 2, e é também mais baixo do que o valor médio encontrado em estudos com a população portuguesa (Castro, Monteiro, Rebelo \& Sá, 1992), o que leva a supor que estas crianças possuirão menor capacidade para lidar com constrangimentos e limitações impostos pela asma, pois como tivemos já oportunidade de referir anteriormente, a auto-estima tem vindo a ser apontada como um factor protector na exposição ao risco (Rutter, 1992). Relativamente ao coping, as crianças que constituem este perfil apresentam valores mais elevados de 
frequência e eficácia, o que de acordo com a literatura, parece indicar que possuem mais recursos para lidar com os problemas com que se confrontam. No entanto, é interessante verificar que estas crianças são as que apresentam valores mais baixos de qualidade de vida e valores mais altos de depressão/ansiedade. Ou seja, apesar de parecem mais equipadas para lidar com stressores, estas crianças revelam mais sintomas de mal-estar a nível emocional e percepcionam a sua qualidade de vida como menos elevada. Para complementar a caracterização do perfil, iremos agora referir os resultados obtidos através da análise de diferenças entre os clusters com base nas variáveis que não foram utilizadas para a sua determinação. Uma destas variáveis foi a das habilidades sociais, um dos três indicadores de adaptação, relativamente à qual apenas dispúnhamos de dados relativos a 63 crianças. Quanto a este indicador, as crianças que integram este perfil (1) também apresentam os valores mais baixos, o que está em consonância com os valores dos outros indicadores.

No que diz respeito às variáveis relativas à família, é neste cluster que existe maior percentagem de crianças com agregado familiar não intacto, o que poderá contribuir, em parte, para que as crianças não se encontrem tão bem adaptadas. Como referimos anteriormente, a investigação tem demonstrado que crianças provenientes de famílias onde uma das figuras parentais está ausente se deparam com maiores dificuldades de adaptação, face à presença de uma doença crónica (Brown et al., 2008). Em termos da gestão da doença, é de esperar que um agregado familiar não intacto se depare com maiores dificuldades, quer em termos económicos, quer de organização das tarefas relacionadas com os cuidados a prestar à criança. Quanto ao grau de gravidade da doença, que segundo a literatura revista constitui um risco acrescido para a desadaptação (McQuaid et al., 2001; Everhart \& Fiese, 2008), este perfil integra mais crianças com asma do tipo moderada e menos crianças com asma do tipo ligeiro, ou seja, relativamente ao cluster 2 , inclui mais casos de maior gravidade, pelo que se torna assim compreensivel que estas crianças possuam mais problemas a nivel da adaptação. Ou seja, a combinação de características temperamentais mais desafiantes, de uma menor auto-estima, de um coping mais frequente e percebido como mais eficaz, de uma menor qualidade de vida, de menores habilidades sociais e de mais sintomas de ansiedade/depressão e de uma asma de maior gravidade é entendida como um padrão global que representa um perfil de menor adaptabilidade.

O segundo cluster (2), considerado como sendo o perfil de maior adaptabilidade é constituído pelas crianças com asma que nas variáveis temperamentais apresentam as médias mais elevadas de persistência na tarefa, e mais baixas de reactividade negativa, sociabilidade (retraimento) e actividade, o que de acordo com o que já foi exposto anteriormente, são características que favorecem uma boa adaptação por parte da criança (McClowry, 2002). Ao possuírem padrões comportamentais que são percepcionadas pelos demais como agradáveis e fáceis, estas crianças 
suscitam provavelmente mais interacções positivas por parte destes, o que facilita o seu funcionamento e desenvolvimento em geral. As crianças que integram este cluster são também as que obtiveram valores mais elevados a nível da auto-estima, o que provavelmente se traduzirá por uma maior capacidade para lidar com diferenças de estilo de vida face aos seus pares, que possam ser impostas pela asma, como por exemplo, a impossibilidade de praticar desporto ou de participar em actividades ao ar livre como acampamentos. É constituído pelas crianças que obtiveram valores menos elevados nas variáveis relacionadas com o coping, quer quanto à sua frequência quer quanto à sua eficácia, o que levaria a supor que se trataria de crianças com menos recursos para resolver os seus problemas, se não fossem os valores obtidos a nível dos indicadores de adaptação. Na verdade, estas crianças foram as que apresentaram menos sintomas de ansiedade/depressão e que relataram uma melhor qualidade de vida.

Quanto às restantes variáveis avaliadas após a constituição dos clusters, as crianças que constituem este agrupamento (2) são as que apresentam valores mais elevados de habilidades sociais, resultado que constitui um outro indicador de boa adaptabilidade. Relativamente ao tipo de agregado familiar, as crianças deste perfil são maioritariamente provenientes de agregados intactos, ou seja, constituídos por ambas as figuras parentais, o que parece indicar que possuem um contexto familiar mais favorável a uma melhor adaptação em face da doença. Como já referimos, a família constitui um forte metabolizador do stress associado à gestão de uma doença crónica e sendo capaz de apoiar emocionalmente a criança na vivência de situações menos favoráveis, pode contribuir para diminuir o seu mal-estar e melhorar a sua qualidade de vida (Edwards \& Davis, 1997). O cluster 2 também agrega as crianças com casos menos graves de asma o que, como vimos ao longo deste artigo, parece ser uma condição facilitadora de uma melhor adaptação.

Não foram encontradas diferenças significativas em nenhuma das variáveis sociodemográficas pelo Qui-quadrado (entre os dois clusters), o que vai de encontro a investigações anteriores (Wambolt, Fritz, Mansell, McQuaid, \& Klein, 1998) nas quais se verificou que este tipo de variáveis não estava também associada à adaptação dos sujeitos. Em suma, tendo em conta as características descritas julgamos que o perfil 2 caracteriza as crianças com asma que estão melhor adaptadas ou seja as que demonstraram possuir mais recursos para lidar com sua doença. Tal como sugere a literatura actual, agrupa a maior percentagem de criança da amostra, o que sugere que a maioria das crianças com asma se consegue adaptar bem a esta condição e funcionar dentro dos parâmetros que caracterizam as crianças da população geral.

Quanto ao coping, os resultados merecem-nos algumas reflexões, dado parecem contrariar o que seria esperado do ponto de vista teórico. A literatura geral- 
mente defende que as estratégias de coping constituem um pré-requisito para uma adaptação bem sucedida e que funcionam como um factor protector em situações de risco (Ryan-Wenger et al., 2000). Estas autoras defendem que as crianças com um repertório rico de estratégias de coping, que percepcionam como sendo eficazes para lidar com os seus stressores, constituem um grupo resiliente face a várias situações de adversidade, nomeadamente situações de doença. Tendo em conta a definição de estratégias de coping como sendo comportamentos intencionais e deliberados que envolvem esforço no sentido de lidar com stressores (Lazarus \& Folkman, 1984 cit in Ryan-Wenger, 1990), os resultados encontrados poderão ser interpretados como sendo expressão de que as crianças que mais frequentemente recorrem a estratégias de coping, são aquelas que se confrontam com mais stressores (reais ou imaginados), pelo que são também as que se percepcionam como tendo pior qualidade de vida, que representa uma área de desenvolvimento fundamental para o grupo etário em estudo. Uma outra hipótese de interpretação, será a de considerar que as crianças com uma melhor adaptação, avaliada através de uma melhor qualidade de vida percepcionada, tenham já sido capazes de seleccionar um repertório mais reduzido de estratégias de coping que constituem formas eficazes de lidar com os seus problemas, daí que na medida da frequência de utilização de estratégias de coping obtenham valores menos elevados. Esta interpretação é consistente com a perspectiva de Ryan-Wenger (1990) segundo a qual, à medida que as crianças vão acumulando experiência, vão também recorrendo a um número mais limitado de estratégias.

Como conclusão podemos afirmar que os resultados encontrados mostram que as crianças com asma, enquanto grupo, apresentam características que sugerem maiores dificuldades de adaptação psicológica. No entanto, estes resultados adquirem contornos mais específicos quando os conjugamos com os resultados da análise de clusters. Apesar de a caracterização destas crianças enquanto grupo revelar que estas se distinguem da população em geral, a análise de clusters demonstrou que, enquanto grupo que vive sob uma condição especial, as crianças com asma apresentam alguma variabilidade em termos de adaptação, o que se expressa na identificação de dois perfis de adaptação, que foram interpretados como representando uma situação de maior adaptabilidade e uma outra de pior adaptabilidade, estando a maioria das crianças incluída no perfil que representa a melhor adaptação. O perfil que demonstrou uma maior adaptabilidade era constituído por crianças que apresentavam indicadores de maiores recursos à excepção do coping. Apesar de aparentemente pouco compreensível, este último resultado poderá ser reflexo de um ganho na vivência da asma que se reflecte numa maior sofisticação na utilização das estratégias de coping ou uma menor problematização dos problemas enfrentados pela criança. 
Os resultados reforçam simultaneamente a necessidade de se atender à criança na sua especificidade, dado que, como constatámos, a sua condição de doente se reflecte de forma diferente a nível do seu funcionamento e adaptação, dependendo de características individuais e de recursos que foi adquirindo ao longo da sua trajectória individual. Julgamos ainda que esta investigação pode levantar pistas importantes para a investigação e intervenção não só a nível da asma pediátrica mas também em relação a outros tipos de doença crónica e mesmo a outras situações potencialmente de risco.

\section{Referências bibliográficas}

Achenbach, T.M. (1991). Manual for the Child Behavior Checklist and Revised Child Behavior Profile. Burlington: University of Vermont, Department of Psychiatry.

Aldenderfer, M.S., \& Blashfield, R.K. (1984). Cluster Analysis. London: Sage.

Bacharier, L.B., Boner, a., Carlsen, K-H., Eigenmann, P.A., Frischer, T., Gotz; M. et al. (2008) Diagnosis and treatment of asthma in childhood: a PRACTALL consensus report. Allergy. 63: 5-34.

Barr, R.G., Boyce, W. T. \& Zeltzer, L. K. (1996). The stress-illness association in children: A perspective from the biobehavioral interface. In R., Haggerty, L., Sherrod, N., Garmezy, \& M., Rutter, (Eds) Stress, Risk and Resilience in Children and Adolescents: Processes, Mechanisms, and Interventions. (pp.317-353), Cambridge: Cambridge University Press.

Barros, L. (2003). Psicologia Pediátrica: Perspectiva desenvolvimentista. Lisboa: CLIMEPSI.

Benchell, J., \& Glasgow, A. (1997). The Role of Temperament in Children with Insulin-Dependent Diabetes Mellitus. Journal of Pediatric Psychology, 22(6), 795-809.

Bender, B.G., Annet, R.D., Duttamel, T.R., Rand, C., \& Strunk, R.C. (2000). Relationship between disease and psychological adaptation in children in the Childhood Asthma Management program and their Families. Archives Pediatric Adolescence Medicine, 154(7), 706-713.

Bleil, M.E., Ramesh, S., Miller, B. D., \& Wood, B.L. (2000). The influence of Parent-Child Relatedness on Depressive Symptons in Children with Asthma: Tests of Moderator and Mediator Models. Journal of Pediatric Psychology, 25(7), 481-491.

Borgen,F.H \& Barnett, D.C. (1987). Applying cluster analysis in counselling research. Journal of Counselling Psychology. 34, 456-468.

Brown, R.T., Wiener, L.W., Kupst, M, J.K., Brennan, T., Behrman, R., Compas, B.E. et al. (2008). Single Parenting and Children with Chronic Illness: An Understudied Phenomenon. Journal of Pediatric Psychology.33(4), 408-421.

Carranza, J.A, \& Salinas, C. G. (2003). Temperamento en la infancia: aspectos conceptuales básicos. Barcelona: Ariel Psicología.

Castro, P., Monteiro, M.B., Rebelo \& Sá (1992). Perfil de Auto-percepção para Crianças: estudo de um instrumento de avaliação da auto-estima. Comunicação apresentada no III Simpósio de Investigação Psicológica. Lisboa: Fundação Calouste Gulbenkian.

Carey, W. (2004). Temperament and the Pediatrician. Reprinted from Pediatric Development and Behaviour Online. Consultado a 19 de Julho de 2004 em http.//Www.dppeds. org/articles/detail_p.cfm? id=18. 
Carranza, J.A, \& Salinas, C. G. (2003). Temperamento en la infancia: aspectos conceptuales básicos. Barcelona: Ariel Psicología.

Cichetti, D., \& Toth, S. (1998). Perspectives on Research and Practice in Developmental Psychology. In William Damon (Editor in Chief), I. Siegel, A. Renninger. Child Psychology. Volume Four: Child Psychology in Practice. (pp.479-559). New York: Wiley.

Creer, T.L., \& Bender, B.G. (1995). Pediatric Asthma. In M. Roberts (Eds), Handbook of Pediatric Psychology (pp 219-240). London: Guilford Press.

Edwards, M., \& Davis, H. (1997). Counselling Children with Chronic Medical Conditions. Leicester: British Psychological Society.

Everhart, R.S., Fiese, B.H. (2008). Asthma severity and child quality of life in pediatric asthma: A systematic review, doi:10,1016/j.pec.2008.10.001

Ferreira, H. I. M. M. (2000). A competência social no contexto escolar: um estudo na interface entre a competência social e a realização académica na criança. Dissertação de Mestrado apresentada à Faculdade de Psicologia e Ciências da Educação da Universidade do Porto.

Fonseca, C.F., Simões, A, Rebelo, J.A, Ferreira, J.A \& Cardoso, F. (1994). Um inventário de competências sociais e de problemas de comportamento em crainças e adolescentes - 0 Child Behaviour Checklist de Achenbach. Psychologica, 12, 55-78.

Frankel, K., \& Wamboldt, M. (1998). Chronic Childhood Illness and Maternal Mental Health - Why Should We Care? Journal of Asthma, 35(8), 621-630.

French, D.,J., Christie, M. J. \& West, A. (1994). Quality of life in childhood asthma: development of the Childhood Asthma Questionnaires. In M. Christie \& D. French, (Eds). Assesement of Quality of Life in Childhood Asthma. (pp.157-180) Chur: Harwood Academic Publishers.

Global Initiative for Asthma- GINA (1995). Global strategy for asthma management and prevention. NHLBI/WHO workshop report. National Institutes of Health, National Heart, Lung and Blood Institute, NIH publication number 95-3659.

Gonçalves, M. \& Simões, M.R. (2000). O Modelo Multiaxial de Achenbach (ASEBA) na Avaliação Clínica de Crianças e Adolescentes. In I. Soares (Ed). Psicopatologia do Desenvolvimento: Trajectórias (in) Adaptativas ao longo da Vida. (pp.11-42). Coimbra: Quarteto.

Gresham, F. M. \& Elliot, S. N. (1984). Assessment and classification of children's social skills: A review of methods and issues. School Psychology Review, 13, 292-301.

Gresham, F. M. \& Elliot, S. N. (1990). Social Skills Rating system: Manual. Circle Pines, MN: American Guidance Service.

Hérnandez, M. J. (1995). Aspectos básicos da Psicopatologia Infantil. In M.J. Hérnadez (Ed). Psicopatologia Infantil. (pp.19-35). Granada: Ediciones Aljibe.

Isaacs, D. \& Sewell, J.R. (2003). Children with chronic conditions. MJA, 179(5),235-236.

Kaugars, A.S., Klinnert, M.D., \& Bender, B.G. (2004). Family Influences on Pediatric Asthma. Journal of Pediatric Psychology, 29(7),475-491.

Kim, S.P., Ferrara, A., Chess, S. (1980). Temperament of asthmatic children. A preliminary study. Journal of Pediatrics, 97(3), 483-486.

Klinnert, M., McQuaid, E., McCormick, D., Adinoff, A., \& Bruant, N. (2000). A Multimethod Assessement of Behavioral and Emotional Adjustment in Children with Asthma. Journal of Pediatric Psychology, 25(1), 35-47.

Lehrer, P.M., Feldman, J., Giardino, N., Song, H-S., \& Schamaling, K. (2002) Psychological aspects of asthma. Journal of Consulting and Clinical Psychology. 70(3), 691 
Lemanek, K., \& Hood, C. (1999). Asthma. In Ronald. T. Brown. Cognitive Aspects of Chronic Illness in Children. (pp.78-104). London: Guilford Press.

Lemos, M. S. \& Meneses, H. I. (2002). A avaliação da competência social: Versão Portuguesa da Forma para professores do SSRS. Psicologia, Teoria e Pesquisa, 18(3),167-274.

Lima, L., Lemos, M. S. \& Guerra (2002). Estudo das qualidades psicométricas do SCSI

(Schoolagers' Coping Strategies Inventory) numa população portuguesa. Análise psicológica, $4(\mathrm{XX})$, 555-570.

Lima, L., Guerra, P.M. \& Lemos, M.S. (2009). Adaptação da escala genérica do Inventário Pediátrico de Qualidade de vida - Pediatric Quality of Life Inventory 4.0 - PedsQL, a uma população portuguesa. Revista Portuguesa de Saúde Pública. Volume temático, 8, 83-95

Lima, L., Lemos, M. S. \& Guerra, M.P. (2010). Adaptação do inventário de temperamento para crianças em idade escolar - SATI a uma população portuguesa. Psicologia, Saúde e Doenças. 11(1), 51-66

Liu, C. \& Wan, G. (2001). The study of temperament in children with asthma. CJCP, 9(3),182-183.

Masten, A.S., Morison, P., Pellegrini, D. \& Tellegen, A. (1992). Competence under stress: risk and protective factors. In J. Rolf, A. Masten, D. Ciccheti, K, Nuechterlein \& S. Weintraub,. (Eds) Risk and Protective Factors in the Development of Psychopathology (pp.236-256). Cambridge: Cambridge University Press.

McClowry, S.G. (2002). The temperament Profiles of School Age Children. Journal of Pediatric Nursing, 17(1),3-10.

McQuaid, E.L., Kopel, S.J., \& Nassau, J.H. (2001). Behavioral Adjustment in children with asthma : A meta-analysis. Journal of Developmental and Behavioral Pediatrics; 22(6), 430-439.

McQuaid, E. \& Walders, N. (2003). Pediatric Asthma. In M. Roberts (Eds), Handbook of Pediatric Psychology (pp 269-285). London: Guilford Press.

Moran, M. G. (2000) Doenças Pulmonares e Reumatológicas. In A. Stoudemire (org). Fatores Psicológicos Afetando Condições Médicas. (pp. 143-158). Porto Alegre: Artes Médicas Sul.

Pauli-Pott, U., Darui, A., \& Beckman, D. (1999). Infants with Atopic Dermatitis : Maternal Hopelessness, Child-Rearing Attitudes and Perceived Infant Temperament. Psychotherapy and Psychosomatics, 68:39-45.

Pestana, M. H., \& Gageiro, J. N. (2000). Análise de dados para Ciências Sociais. A Complementaridade do SPSS. Lisboa: Edições Sílabo.

Rothbart, M., \& Bates, J. (1998). Temperament. In W. Damon \& N. Eisenberg (Eds), Handbook of child psychology-Volume 3: Social, emotional and Personality development (pp.105-164). New York: Jonh Willey \& Sons.

Rutter, M. (1992). Psychosocial resilience and protective mechanisms. In J. Rolf, A. Masten, D. Ciccheti, K, Nuechterlein \& S. Weintraub,. (Eds) Risk and Protective Factors in the Development of Psychopathology (pp.181-214). Cambridge: Cambridge University Press.

Ryan-Wenger, N.(1990). Development and psychometric properties of the schoolagers' coping strategies inventory. Nursing Research, 39(6), 344-349.

Ryan-Wenger, N. (1996). Children, Coping and the stress of Illness: a synthesis of the research. Journal of The Society of Pediatric Nurses, 1(3) 126-139.

Schuman, W., \& La Greca, A. (1999). Social Correlates of Chronic Disease. In R. T. Brown (Eds.), Cognitive Aspects of Chronic Illness in Children. (pp. 289-311). London: Guilford Press. 
Smeekens, S., Riksen-Walraven, J., \& Bakel, H. (2008). Profiles of competence and adaptation in preschoolers as related to the quality of parent-child interaction. Journal of Research in Personality, 42(6), 1490-1499.

Sharp, S., \& Cowie, H. (1998). Counselling and Supporting Children in Distress. London: Sage. Simões, M. M. R. (2000). Investigações no âmbito da Aferição Nacional do Teste das Matrizes Progressivas Coloridas de Raven (M.P.C.R.). Coimbra: Fundação Calouste Gulbenkian e Fundação para a Ciência e Tecnologia.

Spirito, A., Stark, L.J., Gil, K.M., \& Tyc, V.L. (1995). Coping with everyday and disease-related by chronically ill children and adolescents. Journal of The American Academy of Child and Adolescent Psychiatry, 34(3), 283-291.

Thompson, R.J., \& Gustafson, K. E. (1996). Adaptation to Childhood Childhood IIIness. Washington: American Psychological Association.

Thompson, R. J., Gustafson, K. E., George, L. K., \& Spock, A.. (1994). Change Over a 12-Month Period in the Psychological Adjustment of Children with Cystic Fibrosis. Journal of Pediatric Psychology, 19(2), 189-203.

Varni, J.W., Seid, M., \& Kurtin, P.S. (2001) The PedsQL 4.O: Reliability and validity of the Pediatric Quality of Lfe Inventory 4.0 version, Medical Care, 39,800-812.

Vinson, J.A. (2002). Children With Asthma : Initial Development of The Child Resilience Model. Pediatric Nursing, 28(2), 149-158.

Wallander, J., \& Varni, J. (1998). Effects of Pediatric Physical Disorders on Child and Family Adjustment. Journal Child Psychology Psychiatry, 39 (1), 29-46.

Wamboldt, M.Z., Fritz, G., Mansell, A., McQuaid, E.L., \& Klein, R.B. (1998). Relationship of asthma severity and psychological problems in children. Journal of The American Academy of Child and Adolescent Psychiatry, 37(9), 943-951.

World Health Organization (2000). Bronchial Asthma. WHO Fact Sheet $n^{\circ} 206$. Revised January.

\section{Profiles of pychological adjustment of children with asthma}

This study aims at the characterization of children with asthma and the identification of patterns of psychological adjustment. The sample consisted of 89 children, from both sexes and aged between 8 and 12 years old with physician's diagnosed asthma. Measures of temperament, intelligence, self-esteem, frequency and efficacy of coping strategies, symptoms of anxiety/depression, social abilities and generic quality of life were used and cluster analysis were conducted.

The results found suggest that children with asthma, as a group, have characteristics that imply greater difficulties in terms of psychological adaptation. Nevertheless cluster analysis revealed the existence of two distinct profiles in terms of adjustment and showed that although asthma represents a risk of psychological desaptation it is possible to cope positively wth the disease.

KEY-WORDS: Asthma; Patterns of psychological adjustment; Cluster analysis. 


\section{Profils d'adaptation psychologique de l'enfant avec asthme}

L'objectif principal de cette étude consiste à contribuer à la compréhension de l'impact de l'asthme dans l'adaptation de l'enfant. On a essayé de caractériser d'un point de vue psychologique les enfants avec asthme e leurs familles, et d'identifier différents profils d'adaptation.

L'échantillon était constitué de 89 enfants des deux sexes, d'âges compris entre 8 et 12 ans avec d'asthme. En ce qui concerne les enfants, on a évalué les dimensions du tempérament, de l'intelligence, de l'estime de soi, de stratégies de coping et du nombre de situations de stress vécues pendant l'année précédente. Pour la famille, on a fait l'évaluation du type de groupe familial, l'humeur de la mère et les altérations et interférences provoquées par l'asthme dans les routines familiales. Trois variables ont été sélectionnées comme indicateurs d'adaptation: symptômes d'anxiété et dépression, les compétences sociales et la qualité de vie.

Les résultats de l'étude montrent que les enfants asthmatiques présentent deux profils d'adaptation différents qu'evidencient la possibilité de faire face à la maladie.

MOTS-CLÉS: Asthme, Profils d'adaptation psychologique, Cluster analysis. 